\title{
OPEN Deep radiomics-based survival prediction in patients with chronic obstructive pulmonary disease
}

\author{
Jihye Yun ${ }^{1}$, Young Hoon Cho ${ }^{2}$, Sang Min Lee ${ }^{1}$, Jeongeun Hwang ${ }^{3}$, Jae Seung Lee ${ }^{4}$, \\ Yeon-Mok Oh${ }^{4}$, Sang-Do Lee ${ }^{4}$, Li-Cher Loh ${ }^{5}$, Choo-Khoon Ong ${ }^{5}$, Joon Beom Seo ${ }^{1 \bowtie}$ \& \\ Namkug Kim ${ }^{1,6 \bowtie}$
}

Heterogeneous clinical manifestations and progression of chronic obstructive pulmonary disease (COPD) affect patient health risk assessment, stratification, and management. Pulmonary function tests are used to diagnose and classify the severity of COPD, but they cannot fully represent the type or range of pathophysiologic abnormalities of the disease. To evaluate whether deep radiomics from chest computed tomography (CT) images can predict mortality in patients with COPD, we designed a convolutional neural network (CNN) model for extracting representative features from CT images and then performed random survival forest to predict survival in COPD patients. We trained CNNbased binary classifier based on six-minute walk distance results ( $>440 \mathrm{~m}$ or not) and extracted highthroughput image features (i.e., deep radiomics) directly from the last fully connected layer of it. The various sizes of fully connected layers and combinations of deep features were experimented using a discovery cohort with 344 patients from the Korean Obstructive Lung Disease cohort and an external validation cohort with 102 patients from Penang General Hospital in Malaysia. In the integrative analysis of discovery and external validation cohorts, with combining 256 deep features from the coronal slice of the vertebral body and two sagittal slices of the left/right lung, deep radiomics for survival prediction achieved concordance indices of $0.8008(95 \% \mathrm{Cl}, 0.7642-0.8373)$ and $0.7156(95 \%$ $\mathrm{Cl}, 0.7024-0.7288)$, respectively. Deep radiomics from $\mathrm{CT}$ images could be used to predict mortality in COPD patients.

Chronic obstructive pulmonary disease (COPD) is a chronic inflammatory lung disease that causes airflow limitation and symptoms include shortness of breath, frequent coughing or wheezing, and excess mucus (sputum) production. COPD is a major cause of chronic morbidity and mortality throughout the world; many people suffer from this disease for years and die prematurely from it or its complications ${ }^{1}$. Pulmonary function tests (PFTs) are currently used to diagnose and classify the severity of COPD, but they cannot fully represent the type and range of pathophysiological abnormalities of the disease. In particular, PFTs tend to be relatively insensitive to early COPD symptoms and subtle symptom changes ${ }^{2}$. Furthermore, patients with similar PFT values may exhibit completely different clinical and radiologic phenotypes.

Medical imaging, which provides multiparametric morphologic and functional information, plays an increasingly significant role in precision medicine. Chest computed tomography (CT) provides in vivo visual information that can be used to investigate structural and underlying pathophysiologic changes in COPD patients, and thus allows analysis of primary features of COPD including morphologic characteristics and the distribution of both emphysema and small airway disease ${ }^{3-6}$. However, qualitative CT assessment by radiologists, which has been the mainstream method for acquiring information from CT scans, is prone to inter-reader, and sometimes even intra-reader, variability, limiting its application to broad clinical and experimental settings ${ }^{7}$. Therefore, the need for more objective CT-based measures has grown significantly.

\footnotetext{
${ }^{1}$ Department of Radiology, Asan Medical Center, University of Ulsan College of Medicine, Seoul, South Korea. '2Department of Radiology, Korea University Guro Hospital, Korea University College of Medicine, Seoul, South Korea. ${ }^{3}$ Department of Medicine, University of Ulsan College of Medicine, Seoul, South Korea. ${ }^{4}$ Department of Pulmonary and Critical Care Medicine and Clinical Research Center for Chronic Obstructive Airway Diseases, Asan Medical Center, University of Ulsan College of Medicine, Seoul, South Korea. ${ }^{5}$ Department of Medicine, RCSI \& UCD Malaysia Campus, Penang, Malaysia. ${ }^{6}$ Department of Convergence Medicine, Asan Medical Institute of Convergence Science and Technology, Asan Medical Center, University of Ulsan College of Medicine, Seoul, South Korea.『email: seojb@amc.seoul.kr; namkugkim@gmail.com
} 
Radiomics has been proposed to explore the correlation among medical images, other -omics, and clinical parameters, and interest in its application has been growing since it has the potential to provide significant interpretive and predictive information to support decision making. Radiomics is the process of extracting high-throughput quantitative features from radiographic images and building predictive models relating image features to genomic patterns and clinical outcomes ${ }^{8}$. In the past few years, a number of radiomics models have been proposed for tumor classification ${ }^{9-11}$, survival prediction ${ }^{12,13}$, and recurrence prediction ${ }^{14,15}$.

In radiomics-based analysis, high-throughput feature extraction (i.e., radiomics) is a critical task. In previous studies, most extracted features were designed by hand or explicitly. In the field of COPD, quantitative CT imaging methods have been proposed to provide more precise and reproducible estimates of the severity and distribution of emphysema and airway disease ${ }^{16}$. In the early days of quantitative imaging biomarkers, research was weighted toward quantification of emphysema, and there has recently been an increasing number of publications targeting the airway component of COPD, which includes direct airway parameter measurements and quantification of air trapping as functional manifestations of small airway disease ${ }^{17}$. Moreover, quantitative pulmonary vascular features turned out to be associated with COPD severity and emphysema extent ${ }^{18}$. Although the number of handcrafted features can reach tens of thousands, these features are shallow and low order. They may not fully characterize image heterogeneity and may limit the potential of radiomics models. Therefore, it is necessary to assess deeper and higher-order features that may improve the predictive performance of radiomics models.

Recently, the performance of deep learning has been intensively demonstrated in computer vision. In particular, a convolutional neural network (CNN), which uses a trainable filter bank with an extensive weight-sharing scheme, can quickly outperform state-of-the-art approaches in many computer vision tasks, including image classification and segmentation ${ }^{19-22}$. These deep learning-based approaches have also impressive results for CT analysis in $\mathrm{COPD}^{23}$.

CNN can be incorporated into current radiomics models by extracting many deep features from hidden layers ${ }^{24,25}$. These deep features, extracted not by feature engineering (handcrafting) but by feature learning, could contain more representative and high-level medical image information and provide more predictive patterns compared to handcrafted features. In this paper, we propose a deep feature-based radiomics model for predicting the overall survival of COPD patients.

\section{Materials and methods}

Patients. There were two groups of patients: (1) a discovery cohort with 344 patients from the Korean Obstructive Lung Disease (KOLD) cohort and (2) an external validation cohort with 102 patients from Penang General Hospital in Malaysia. The inclusion and exclusion criteria for the discovery cohort have been published previously ${ }^{26}$. In short, patients over 18 years of age with chronic respiratory symptoms and airflow limitations or bronchial hyperresponsiveness were included. Between June 2005 and April 2012, 344 patients with an established COPD diagnosis and available volumetric chest CT scans taken at the time of registration were enrolled. Subjects underwent PFTs within two weeks of volumetric chest CT image acquisition. Baseline clinical characteristics, PFT results, six-minute walk distance (6MWD) results, and survival information were documented for all patients. Our institutional review board approved this study, and written informed consent was obtained from all patients. The external validation cohort included patients in the chest clinic of the 1200-bed Penang General Hospital, which is part of the Asian Network of Obstructive Lung Disease ${ }^{27}$. All patients with stable COPD who were referred to or followed up were invited to participate. Prospective data of 112 eligible COPD subjects was available for mortality analysis. Inclusion and exclusion criteria have been published previously ${ }^{28}$. Of 112 patients initially eligible, 10 were excluded due to poor CT image quality. The median follow-up time was 1000 days (range, 60-1400). Quantitative CT and clinical demographic data were collected at the time of study entry. Written informed consent was obtained from all participants. Research and ethical approval was obtained from the National Research and Ethics Committee of Malaysia (NNMR-13-313-15138). All methods were performed in accordance with the relevant guidelines and regulations.

Volumetric chest data acquisition. Discovery cohort volumetric chest CT scans were obtained using 16- or 64-slice multidetector CT (MDCT) scanners produced by two different manufactures (259 CT scans using SOMATOM Sensation 16 or SOMATOM Definition AS from Siemens Healthineers AG, Bonn, Germany; 85 CT scans using Philips Brilliance 16, 40, or 64 from Philips Medical Systems, Best, Netherlands). Patients were scanned craniocaudally in the supine position during full inspiration. Routine administration of intravenous contrast media was not required for image acquisition using either type of scanner. CT scan parameters were: collimation of $16 \times 0.75 \mathrm{~mm}$, effective mAs of $100, \mathrm{kVp}$ of 140 , and pitch of 1 . CT data were reconstructed at a 0.75- $\mathrm{mm}$ slice thickness and 0.7- $\mathrm{mm}$ increment using a B30f kernel for Siemens scanners and a 0.8-mm slice thickness and $0.8-\mathrm{mm}$ increment using a standard reconstruction algorithm for Philips scanners.

External validation cohort volumetric chest CT scans were obtained using a 64-slice MDCT scanner (SOMATOM Sensation 64; Siemens Healthineers AG, Forchheim, Germany) at the Loh Guan Lye Specialist Centre in Penang, Malaysia. CT scans were obtained using standardized protocol from the Research Institute of Radiology of the Asan Medical Center in Seoul, South Korea ${ }^{4,29}$. The CT scan parameters were a collimation of $0.75 \mathrm{~mm}$, effective $\mathrm{mAs}$ of $100, \mathrm{kVp}$ of 140 , and pitch of 1 . Patients were scanned craniocaudally in the supine position during full inspiration. Images were reconstructed using a soft kernel (B30f; Siemens Healthineers AG) from thoracic inlet to lung base. Image quality and protocol compliance were verified by the Asan Medical Center.

Deep features extraction. We trained a CNN model to obtain high-level representative information from medical images, and high-throughput image features (i.e., deep radiomics) were directly extracted from the last fully connected layer. The CNN performed binary classification based on 6MWD testing, one of the most impor- 


\begin{tabular}{|c|c|c|c|c|c|c|}
\hline & \multicolumn{4}{|l|}{ Discovery cohort } & \multirow[b]{2}{*}{$\begin{array}{l}\text { External } \\
\text { validation cohort }\end{array}$} & \multirow{2}{*}{$\begin{array}{l}p \text {-value } \\
\text { (discovery vs } \\
\text { external } \\
\text { validation } \\
\text { cohort) }\end{array}$} \\
\hline & $6 \mathrm{MWD}>440 \mathrm{~m}$ & $6 \mathrm{MWD} \leq 440 \mathrm{~m}$ & $\begin{array}{l}p \text {-value }(>440 \mathrm{~m} \\
\text { vs. } \leq 440 \mathrm{~m})\end{array}$ & Total & & \\
\hline Patients (N) & 157 & 187 & & 344 & 102 & \\
\hline $\operatorname{Mortality}^{\star}(\mathrm{N})$ & $12(7.6 \%)$ & $31(16.6 \%)$ & 0.018 & $43(12.5 \%)$ & $18(17.6 \%)$ & 0.185 \\
\hline $\begin{array}{l}\begin{array}{l}\text { Follow-up } \\
\text { (months) }\end{array} \\
\end{array}$ & $84.8(2,149)$ & $57.1(2,148)$ & $<0.001$ & $69.8(2,149)$ & $32.9(2,47)$ & $<0.001$ \\
\hline Age (years) & $64.0 \pm 7.0$ & $69.8 \pm 7.4$ & $<0.001$ & \begin{tabular}{|l|}
$67.2 \pm 7.8$ \\
\end{tabular} & $68.3 \pm 8.0$ & 0.601 \\
\hline Gender & & & 0.151 & & & 0.006 \\
\hline Male (N) & $156(99.4 \%)$ & $182(97.3 \%)$ & & $338(98.3 \%)$ & $95(93.1 \%)$ & \\
\hline Female $(\mathrm{N})$ & $1(0.6 \%)$ & $5(2.7 \%)$ & & $6(1.7 \%)$ & $7(6.9 \%)$ & \\
\hline Height & $167.0 \pm 5.4$ & $164.4 \pm 6.3$ & $<0.001$ & $165.6 \pm 6.1$ & - & \\
\hline \begin{tabular}{|l|} 
Weight \\
\end{tabular} & $66.0 \pm 8.7$ & $61.2 \pm 10.1$ & $<0.001$ & $63.4 \pm 9.8$ & - & \\
\hline 6MWD (meters) ${ }^{* *}$ & $499.7(441,652)$ & $368.8(120,440)$ & $<0.001$ & $428.6(120,652)$ & - & \\
\hline \multicolumn{7}{|l|}{ PFT } \\
\hline $\begin{array}{l}\mathrm{FEV}_{1}(\% \text { pre- } \\
\text { dicted) }\end{array}$ & $57.9 \pm 16.5$ & $53.5 \pm 18.0$ & 0.022 & $55.5 \pm 17.4$ & $47.9 \pm 21.3$ & $<0.001$ \\
\hline FVC (\% predicted) & $87.5 \pm 17.4$ & $84.6 \pm 17.8$ & 0.137 & $85.9 \pm 17.7$ & $65.1 \pm 22.9$ & $<0.001$ \\
\hline $\mathrm{FEV}_{1} / \mathrm{FVC}$ & $47.7 \pm 9.8$ & $43.9 \pm 11.3$ & 0.001 & $45.7 \pm 10.8$ & $53.0 \pm 11.1$ & $<0.001$ \\
\hline Gold stage & & & $<0.001$ & & & $<0.001$ \\
\hline Stage I (N) & $23(14.7 \%)$ & $13(7.0 \%)$ & & $36(10.5 \%)$ & $5(4.9 \%)$ & \\
\hline Stage II (N) & $84(53.5 \%)$ & $87(46.5 \%)$ & & $171(49.7 \%)$ & $37(36.3 \%)$ & \\
\hline Stage III (N) & $44(28.0 \%)$ & $70(37.4 \%)$ & & $114(33.1 \%)$ & $42(41.2 \%)$ & \\
\hline Stage IV $(\mathrm{N})$ & $6(3.8 \%)$ & $17(9.1 \%)$ & & $23(6.7 \%)$ & $18(17.6 \%)$ & \\
\hline
\end{tabular}

Table 1. Baseline clinical characteristics and mortality data. Continuous variables are presented as mean \pm standard deviation and categorical data are presented as the number of patients with percentages in parentheses. 'Follow-up' and '6MWD' are presented as the mean with minimum and maximum values in parentheses. $6 M W D$ six-minute walk distance, $P F T$ pulmonary function test, $F E V_{1}$ forced expiratory volume in $1 \mathrm{~s}, F V C$ forced vital capacity, GOLD Global Initiative for Chronic Obstructive Lung Disease. ${ }^{\star}$ Overall survival was investigated as study end-point, which is defined as the time until death from any cause. ${ }^{* *} 6 \mathrm{MWD}$ information was only in the discovery cohort, and Supplementary Figure S2 shows the distribution of it.

tant factors for evaluating the ability to perform activities of daily living. According to the 2015 European Society of Cardiology/European Respiratory Society Guidelines for diagnosing and treating pulmonary hypertension, a $6 \mathrm{MWD}$ result $>440 \mathrm{~m}$ is one of several factors associated with low one-year mortality ${ }^{30}$, so $440 \mathrm{~m}$ was selected as the optimal threshold. Therefore, training the classifier with deep learning using 6MWD results $>440 \mathrm{~m}$ could not only reveal known prognostic features but also potentially identify previously unknown ones. With the value of $440 \mathrm{~m}$, the discovery cohort consisted of 157 patients with 6MWD over $440 \mathrm{~m}$ and 187 patients with $6 \mathrm{MWD}$ less than $440 \mathrm{~m}$, not causing data imbalance in binary classification. The baseline clinical characteristics and the Kaplan-Meier-estimated cumulative survivals of these two groups were compared in Table 1 and Fig. 1, respectively, and a CNN-based binary classifier was designed to distinguish these two groups. Due to the nature of the discovery cohort and the limited processing capabilities of existing graphical processing units, the whole slices were not available. Even if it is possible to utilize the whole slices with reduced resolution, there should be a sufficient number of datasets to be able to train them. Considering the number and mortality of our dataset, training the whole slices was difficult. So, with the advices of expert radiologists, 11 representative CT slices were selected for each patient based on predetermined anatomic landmarks and used for CNN input: (1) three coronal slices at the vertebral body, center of the tracheal carina, and superior vena cava, (2) two sagittal slices at the center of the left and right lung, and (3) six axial slices (two uppers, center and three lowers) at 2-cm intervals at the center of the tracheal carina (Fig. 2). Anatomic landmarks were selected via consensus of two thoracic radiologists (each with more than 20 years of experience) with the idea that information on the distribution of emphysema or airway changes should be necessary, and $11 \mathrm{CT}$ slices in both cohorts were manually selected by an experienced research assistant, and then finally confirmed by a thoracic radiologist. In the case of axial slices, $2-\mathrm{cm}$ intervals were adopted as a way of obtaining the maximum information with a small number of slices. To classify low-risk patients based on 6MWD results, the CNN was designed to have five convolution blocks and one fully connected layer (Fig. 3). Each convolution block consisted of a convolutional layer with 32 learnable filters followed by batch normalization, rectified linear unit activation, and max-pooling. To prevent overfitting, a connection dropout probability of 0.5 was added to the fully connected layer. Finally, low-risk probabilities were calculated using the softmax function. Using the same CNN architecture, 11 models were separately trained with each of the 11 selected slices and then used to extract deep features from each image. The performances of 11 models were validated using five-fold cross-validation (Supplementary Table S1). We could not verify them with the external validation cohort since there was no 6MWD information, but performance can be inferred 


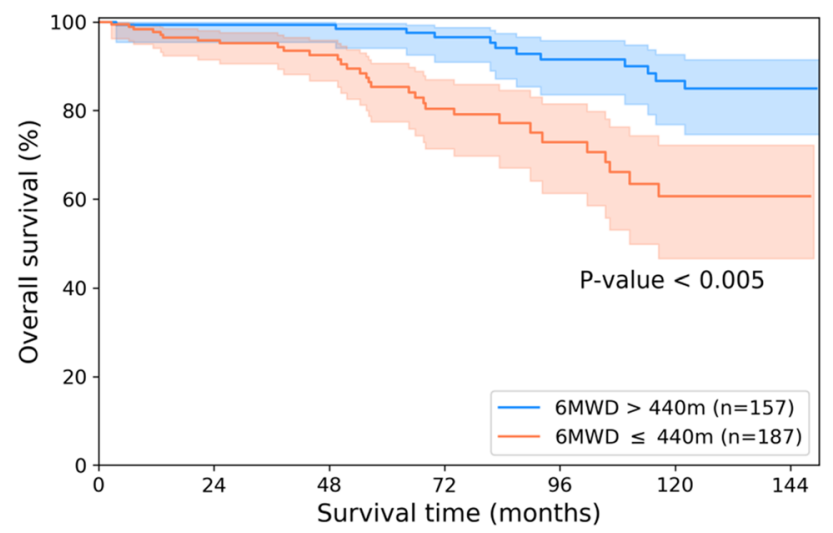

Figure 1. Kaplan-Meier survival curves for patients with $6 \mathrm{MWD}$ over and less than $440 \mathrm{~m}$ in discovery cohorts. We designed and trained a CNN-based binary classifier to obtain high-level representative information from chest CT that can predict mortality in patients with COPD. This CNN-based binary classifier was based on the 6MWD testing results, and the discovery cohort was divided into two groups with the value of $440 \mathrm{~m}$ : 157 low-risk patients and high-risk 187 patients. The estimated cumulative survivals of these two groups were significantly different.

by whether our survival prediction model works well. Nevertheless, the fact that our CNN models for extracting deep features have not been tested well enough to prove their performances is a limitation of our model. Deep features were obtained by normalizing the information of the last fully connected layer; we designed and compared models with various sizes of fully connected layers of 128, 256, 512, and 1024. The CNN designed for extracting deep features was implemented in Keras with a Theano backend.

Survival analysis and statistical comparison. To predict overall survival in COPD patients, random survival forest (RSF) with deep features of each slice was performed. Our deep features were predominantly black box features so that it was difficult to effectively reduce multiple collinear and correlated predictors that could produce unstable estimates and might overfit predictions. RSF is a censored data extension of the Random Forest method, where the ensemble survival function is constructed by aggregating tree-based estimator ${ }^{31}$. We expected that RSF would effectively ensemble the features extracted from our censored data to predict mortality. RSF analysis was implemented using the randomForestSRC R package with default settings. RSF calculated a survival curve for each patient, grew a forest using log-rank splitting, and then averaged the results of the forest, obtaining a stable result. Using RSF, we could predict the survival and cumulative hazard function of individuals. The performance of the proposed deep radiomics-based survival prediction model was evaluated in two independent datasets: (1) the discovery cohort $\left(\mathrm{KOLD}^{26}\right)$ and (2) the external validation cohort (Malaysia $\left.{ }^{28}\right)$. For a quantified comparison, we computed the concordance probability (C-index) and time-dependent area under the receiver operating characteristic (ROC) curve (AUC). C-index is the frequency of concordant pairs among all pairs of subjects and can be used to measure and compare the discriminative power of a risk prediction model. The time-dependent AUC has incorporated time dependency in AUC in time-event data for individuals instead of using the standard ROC curve approach ${ }^{32,33}$, dealing with censored data and yielding different values of AUC at each time point. Internal validation used ROC curves for 3- and 5-year survival, but external validation was not calculated time-dependent AUC because of its follow-up duration. The time-dependent AUC was implemented using the timeROC R package.

\section{Results}

There were two groups of patients: (1) a discovery cohort with 344 patients from KOLD cohort ${ }^{26}$ and (2) an external validation cohort with 102 patients from Penang General Hospital in Malaysia ${ }^{28}$. Baseline clinical characteristics and mortality data are summarized in Table 1 . Overall survival was investigated as the study end-point, which is defined as the time until death from any cause, and Supplementary Figure S1 shows the Kaplan-Meier survival curves of two cohorts.

Internal validation. Deep feature-based survival analysis was performed via five-fold cross-validation of the discovery cohort to determine which features provide more high-level medical image information and predictive patterns. In order to find the optimal number of features suitable for predicting overall survival in COPD patients, we trained the binary classifier of $6 \mathrm{MWD}$ test results with various sizes of fully connected layers of 128 , $256,512,1024$, and then extracted deep features using the representative $11 \mathrm{CT}$ slices. Mortality prediction performance was evaluated using combinations of deep features of each slice, which were obtained by pooling (e.g., for the combination of $\mathrm{C} 1$ and $\mathrm{C} 2$ with 256 deep features, 512 deep features were used as the input to the RSF). We performed RSF for all combinations of 11 slices. Depending on the number of selected samples, the following numbers of combinations were produced, and RSF was performed for a total of 2047 combinations: 1- and 10 -selection, 11 combinations; 2 - and 9-selection, 55 combinations; 3 - and 8-selection, 165 combinations; 4- and 


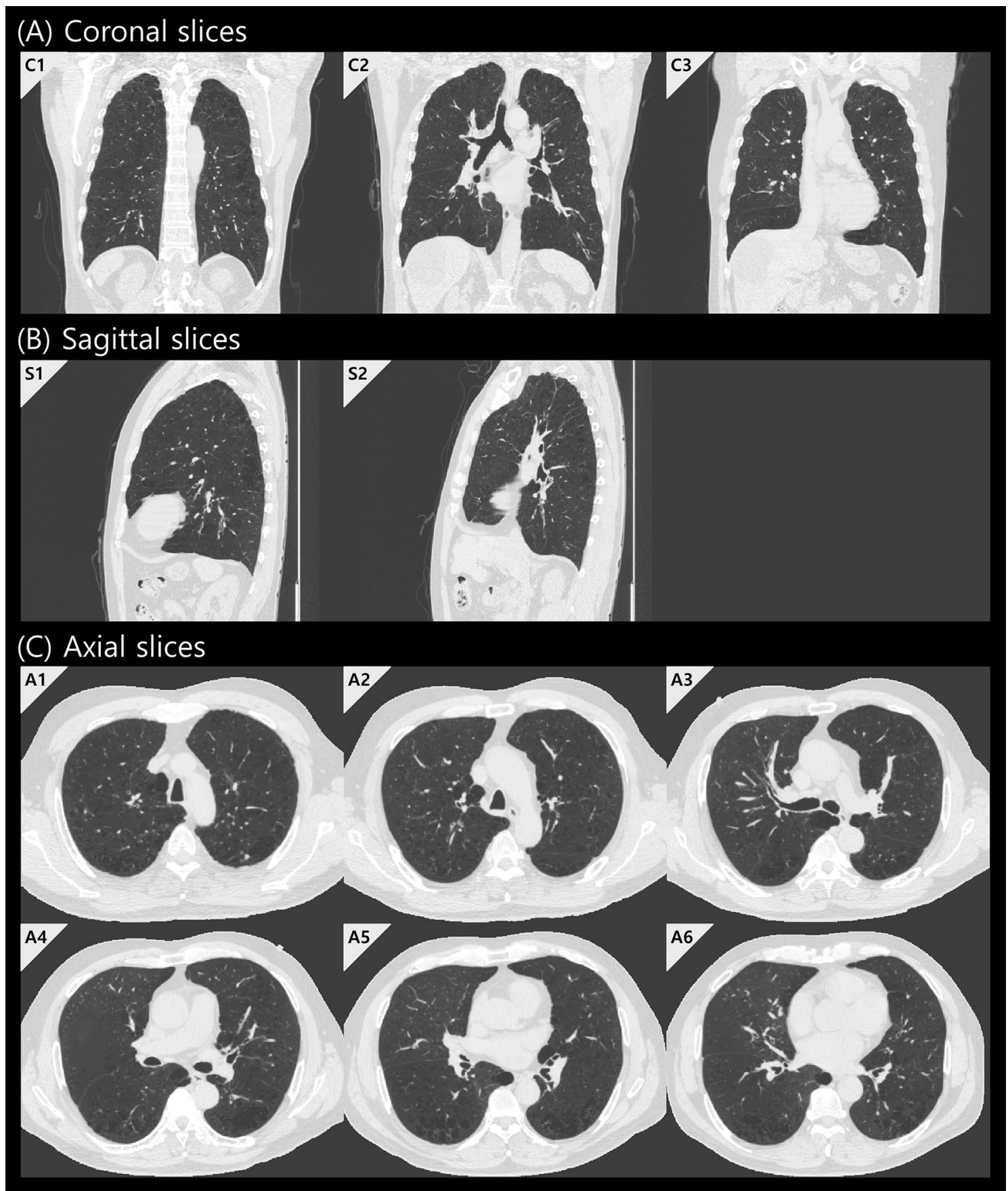

Figure 2. Convolutional neural network $(\mathrm{CNN})$ model input to extract deep features. To extract deep features, 11 computed tomography slices were selected for $\mathrm{CNN}$ model input based on predetermined anatomic landmarks. (A) Coronal slices at the vertebral body (C1), center of the tracheal carina (C2), and superior vena cava (C3). (B) Sagittal slices at the center of the left (S1) and right (S2) lung. (C) Axial slices at two upper slices (A1, A2), center slice (A3), and three lower slices (A4, A5, A6) at 2-cm intervals at the center of the tracheal carina.

7-selection, 330 combinations; 5- and 6-selection, 462 combinations; 11 -selection, 1 combination. The number of $k$-element ( $k$-selection) combinations of $n$ objects without repetition is ${ }_{n} C_{k}=n ! / k !(n-k)$ !-for example, 2- and 9-selection lead to ${ }_{11} C_{2}={ }_{11} C_{9}=11 ! / 2 ! 9 !=55$ distinct combinations. Eventually, the experiments were performed with size of deep features of 128, 256, 512, and 1024, and the performance of all combinations of 11 representative CT slices in each size of deep features was compared and top 5 combinations were summarized in Table 2. The performance of 256 deep features was superior, and the highest C-index of 0.8008 (95\% CI, 0.7642-0.8373) was obtained by combining the coronal slice of the vertebral body and two sagittal slices of the left/right lung $(\mathrm{C} 1+\mathrm{S} 1+\mathrm{S} 2)$. At this combination, AUC for 3- and 5-year survival was and 0.8878 (95\% CI, $0.7900-0.9856)$ and 0.8411 (95\% CI, 0.7901-0.8922), respectively. 


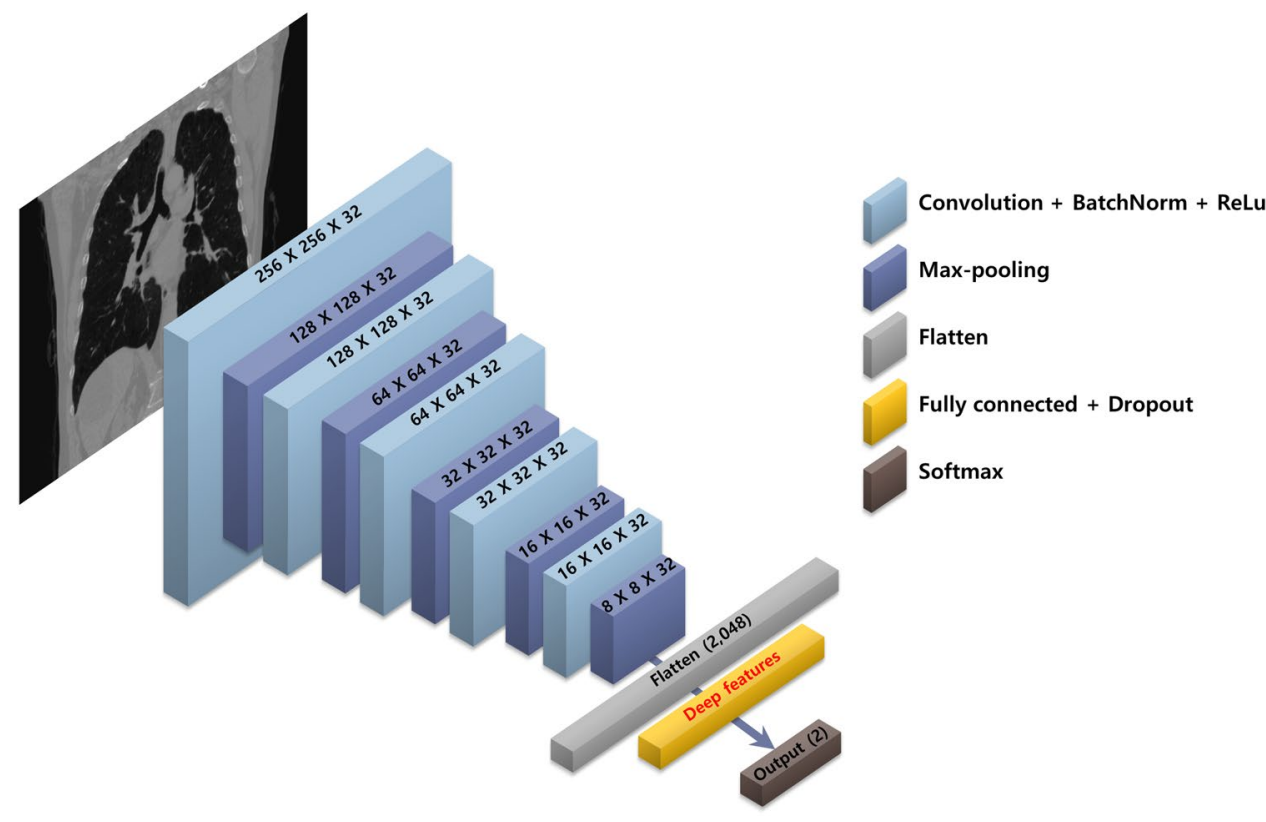

Figure 3. Convolutional neural network $(\mathrm{CNN})$ model architecture. $\mathrm{CNN}$ architecture for classification of six-minute walk distance test results ( $>440 \mathrm{~m}$ or not) to extract deep features. Each of the 11 selected slices was trained separately using the same architecture, which was designed to have five convolution blocks and one fully connected layer. Deep features were obtained by normalizing the information of the last fully connected layer (yellow). Each convolution block consisted of a convolutional layer followed by batch normalization (BatchNorm), rectified linear unit activation (Relu), and max-pooling.

\begin{tabular}{|c|c|c|c|c|c|}
\hline \multirow[b]{2}{*}{$\begin{array}{l}\text { No. of } \\
\text { features }\end{array}$} & \multirow[b]{2}{*}{ Combinations } & \multicolumn{3}{|l|}{ Internal validation } & \multirow{2}{*}{\begin{tabular}{|l|} 
External validation \\
C-index $(95 \% \mathrm{CI})$ \\
\end{tabular}} \\
\hline & & C-index $(95 \%$ CI $)$ & $\begin{array}{l}\text { AUC for 3-year } \\
\text { survival }\end{array}$ & $\begin{array}{l}\text { AUC for 5-year } \\
\text { survival }\end{array}$ & \\
\hline \multirow{5}{*}{128} & 1. $\mathrm{C} 3+\mathrm{S} 2$ & $0.7753(0.7411,0.8095)$ & $0.8042(0.6254,0.9831)$ & $0.8364(0.7895,0.8833)$ & $0.6502(0.6394,0.6610)$ \\
\hline & 2. S2 & $0.7745(0.7237,0.8253)$ & $0.8198(0.6552,0.9844)$ & $0.8197(0.7639,0.8756)$ & $0.6577(0.6466,0.6688)$ \\
\hline & 3. $\mathrm{C} 1+\mathrm{C} 3+\mathrm{S} 2+\mathrm{A} 6$ & $0.7711(0.7210,0.8212)$ & $0.7922(0.5860,0.9984)$ & $0.8247(0.7889,0.8605)$ & $0.6424(0.6268,0.6580)$ \\
\hline & 4. $\mathrm{C} 1+\mathrm{C} 3+\mathrm{S} 2$ & $0.7706(0.7291,0.8121)$ & $0.7928(0.5943,0.9913)$ & $0.8311(0.7850,0.8772)$ & $0.6217(0.6085,0.6348)$ \\
\hline & 5. $\mathrm{C} 2+\mathrm{C} 3+\mathrm{S} 2$ & $0.7676(0.7209,0.8143)$ & $0.8175(0.6404,0.9945)$ & $0.8334(0.7888,0.8779)$ & $0.5803(0.5656,0.5951)$ \\
\hline \multirow{5}{*}{256} & 1. $\mathrm{C} 1+\mathrm{S} 1+\mathrm{S} 2$ & $\begin{array}{l}0.8008(0.7642 \\
0.8373)\end{array}$ & $\begin{array}{l}0.8878(0.7900 \\
0.9856)\end{array}$ & \begin{tabular}{|l|}
$0.8411(0.7901$ \\
$0.8922)$
\end{tabular} & $\begin{array}{l}0.7156(0.7024, \\
0.7288) \\
\end{array}$ \\
\hline & 2. $\mathrm{C} 1+\mathrm{C} 2+\mathrm{S} 1+\mathrm{S} 2+\mathrm{A} 4$ & $0.7959(0.7682,0.8236)$ & $0.8642(0.7638,0.9646)$ & $0.8400(0.7865,0.8935)$ & $0.7130(0.7009,0.7252)$ \\
\hline & 3. $\mathrm{S} 1+\mathrm{S} 2+\mathrm{A} 4+\mathrm{A} 5$ & $0.7948(0.7655,0.8240)$ & $0.8543(0.7638,0.9448)$ & $0.8390(0.7871,0.8954)$ & $0.6742(0.6625,0.6858)$ \\
\hline & 4. $\mathrm{S} 1+\mathrm{S} 2+\mathrm{A} 1+\mathrm{A} 4+\mathrm{A} 6$ & $0.7938(0.7719,0.8157)$ & $0.8337(0.7101,0.9573)$ & $0.8371(0.7819,0.8923)$ & $0.6404(0.6274,0.6533)$ \\
\hline & 5. $\mathrm{S} 1+\mathrm{S} 2+\mathrm{A} 4$ & $0.7930(0.7593,0.8267)$ & $0.8338(0.7259,0.9416)$ & $0.8512(0.8007,0.9017)$ & $0.6400(0.6274,0.6527)$ \\
\hline \multirow{5}{*}{512} & 1. $\mathrm{C} 1+\mathrm{C} 3+\mathrm{S} 2+\mathrm{A} 4$ & $0.7750(0.7432,0.8068)$ & $0.8310(0.7301,0.9319)$ & $0.8254(0.7892,0.8616)$ & $0.6713(0.6600,0.6825)$ \\
\hline & 2. $\mathrm{C} 1+\mathrm{C} 3+\mathrm{S} 1+\mathrm{S} 2+\mathrm{A} 4$ & $0.7716(0.7346,0.8085)$ & $0.8356(0.7027,0.9685)$ & $0.8251(0.7684,0.8817)$ & $0.6714(0.6606,0.6822)$ \\
\hline & $\begin{array}{l}3 . \\
\mathrm{C} 1+\mathrm{C} 3+\mathrm{S} 1+\mathrm{S} 2+\mathrm{A} 1+\mathrm{A} 3\end{array}$ & $0.7659(0.7343,0.7975)$ & $0.8194(0.7260,0.9129)$ & $0.8078(0.7926,0.8230)$ & $0.6277(0.6153,0.6401)$ \\
\hline & $\begin{array}{l}\text { 4. } \mathrm{C} 1+\mathrm{C} 3+\mathrm{S} 1+\mathrm{S} 2+\mathrm{A} 1+ \\
\mathrm{A} 2+\mathrm{A} 3+\mathrm{A} 6\end{array}$ & $0.7657(0.7430,0.7884)$ & $0.8261(0.7294,0.9228)$ & $0.7891(0.7606,0.8177)$ & $0.6660(0.6533,0.6786)$ \\
\hline & 5. $\mathrm{C} 1+\mathrm{C} 3+\mathrm{S} 2$ & $0.7657(0.7250,0.8064)$ & $0.8338(0.7259,0.9416)$ & $0.8238(0.7861,0.8615)$ & $0.6411(0.6273,0.6549)$ \\
\hline \multirow{5}{*}{1024} & 1. $\mathrm{S} 2+\mathrm{A} 2+\mathrm{A} 5$ & $0.7813(0.7333,0.8294)$ & $0.7255(0.5634,0.8877)$ & $0.8257(0.7969,0.8544)$ & $0.6805(0.6186,0.7424)$ \\
\hline & 2. $\mathrm{S} 2+\mathrm{A} 2+\mathrm{A} 5+\mathrm{A} 6$ & $0.7765(0.7439,0.8090)$ & $0.7712(0.6367,0.9056)$ & $0.8094(0.7517,0.8672)$ & $0.6515(0.6024,0.7007)$ \\
\hline & 3. $\mathrm{A} 2+\mathrm{A} 5$ & $0.7744(0.7310,0.8179)$ & $0.7990(0.7072,0.8908)$ & $0.8181(0.7318,0.9045)$ & $0.6305(0.5686,0.6924)$ \\
\hline & 4. $\mathrm{S} 2+\mathrm{A} 2$ & $0.7729(0.7181,0.8278)$ & $0.7864(0.6722,0.9006)$ & $0.8300(0.7413,0.9187)$ & $0.6119(0.5226,0.7013)$ \\
\hline & 5. $\mathrm{C} 1+\mathrm{C} 3+\mathrm{S} 2+\mathrm{A} 5$ & $0.7729(0.7337,0.8120)$ & $0.6881(0.4501,0.9261)$ & $0.8167(0.7563,0.8770)$ & $0.6844(0.6664,0.7023)$ \\
\hline
\end{tabular}

Table 2. Survival prediction performance of deep features in the discovery and external validation cohort. C1, $C 2, C 3$ coronal slices at the vertebral body, center of the tracheal carina, and superior vena cava, respectively; $S 1, S 2$ sagittal slices at the right and left lung, respectively; $A 3$ axial slice at the center of the tracheal carina; $A 1, A 2, A 4, A 5, A 6$ upper two and lower three axial slices at $2-\mathrm{cm}$ intervals at the center of the tracheal carina, respectively. Bold values denote best-performed combinations of each column. 
External validation. The deep radiomics model was evaluated against an external validation cohort (102 CT scans). Top 5 combinations of deep features were used to evaluate the proposed method since they had the best results on internal validation (Table 2). In the external validation, the Rank 1 combination of the internal validation showed also the best C-index of 0.7156 (95\% CI, 0.7024-0.7288).

\section{Discussion}

The current study found that a deep radiomics approach for survival prediction in COPD patients was feasible and showed acceptable performance, which was confirmed by concordant results in an external validation cohort. The deep features from the CNN model using COPD patients' chest CT data were found to be significant and independent predictors of mortality in both the discovery and external validation groups.

Many methodologies using quantitative CT features for quantitative assessment of different COPD components have been studied, but few studies have adapted an integrative approach ${ }^{3,34,35}$. A deep radiomics approach using a CNN to extract many learned features from chest CT images may be helpful in developing clinically useful decision support models.

In this study, a chest CT-based deep radiomics approach with a CNN was used for the first time to predict survival in COPD patients. The CNN performed binary classification based on 6MWD results ( $>440 \mathrm{~m}$ or not). In the discovery and external validation cohorts, with combining the coronal slice of the vertebral body and two sagittal slices of the left/right lung $(\mathrm{C} 1+\mathrm{S} 1+\mathrm{S} 2,256 \times 3$ deep features $)$, deep radiomics for survival prediction achieved C-indices of 0.8008 (95\% CI, 0.7642-0.8373) and 0.7156 (95\% CI, 0.7024-0.7288), respectively, and AUC for 3- and 5-year survival was and 0.8878 (95\% CI, 0.7900-0.9856) and 0.8411 (95\% CI, 0.7901-0.8922), respectively. Comparing performances of the best combination to using all slices for each number of deep features (Supplementary Table S2), it is meaningful to use slices selectively. Among studies using quantitative CT features as a predictor of survival in COPD patients ${ }^{36-38}$, Cho et al ${ }^{38}$ reported the performance using the same datasets of ours. Five features were selected as the final radiomics signature; (1) a percentage of low attenuation area; (2) airway wall thickness of 6th generation bronchus at an internal perimeter of $10 \mathrm{~mm}$; (3) heterogeneity of percentage wall area; (4) heterogeneity of airway wall thickness at an internal perimeter of $10 \mathrm{~mm}$; (5) average pulmonary vessel cross-sectional area measured at $18 \mathrm{~mm}$ from the pleural surface. C-indices of five final radiomics signature were $0.699,0.531,0.615,0.542$, and 0.605 , respectively, and the combinations of radiomics signature were 0.774 . In the same datasets, our deep feature-based survival prediction model outperformed compared to the quantitative CT features. Moll et al. ${ }^{37}$ proposed a survival prediction model using a combination of clinical and quantitative CT features which reported a C-index $\geq 0.7$ and showed 6MWD as the most important predictor. In our discovery cohort, a prediction model with only 6MWD achieved C-index of 0.6072 (95\% CI, 0.6014-0.6130), and our model surpassed it. The mortality prediction performance of our model was externally validated in a separate group of patients. Patient enrollment for the discovery cohort began much earlier (June 2005) than for the external validation cohort (September 2013), which inevitably led to differences in follow-up duration (mean, 69.8 vs. 32.9 months). Although there were some differences in characteristics between two groups including a considerable difference in follow-up duration, external validation was performed to demonstrate the generalizability and transportability of our model.

Both spirometry and multidimensional indices are limited in that they cannot fully represent the type and range of morphologic alterations that may be detectable before functional parameters begin to deteriorate. With a deep radiomics approach, essential information related to phenotypic heterogeneity and pathophysiology may be learned from medical images and used to improve medical decision-making in COPD patients. In the current study, the deep radiomics approach was confined to survival prediction in COPD patients. However, we believe that a deep radiomics approach could potentially be applied to other facets of COPD, such as reliable phenotyping, predicting acute exacerbation, and monitoring treatment response.

The current study is subject to several limitations. First, although our deep feature-based survival prediction model has been integrally analyzed in the discovery and external validation cohorts, a larger study population would have been beneficial, especially in the external validation group. Second, because all patients included in this study were Asian, the results may not be applicable to patients of other ethnicities. However, a major strength of the current research is that the positive discovery group findings were externally validated using a group of patients of a different nationality. That said, further validation of the findings in a larger-scale study with patients of different ethnicities is warranted. Third, the proportion of males in our discovery and external validation cohorts amount to $98.3 \%$ and $93.1 \%$ respectively, resulting in the striking gender imbalance. The imbalance can partly be explained by the cultural environment where the smoking rate in men $(36.7 \%$ in South Korea, and $43.0 \%$ in Malaysia) is overwhelmingly higher than that of women (7.5\% in South Korea, and 1.4\% in Malaysia) in general population ${ }^{39,40}$, and sexual difference in COPD prevalence ${ }^{40-42}$. Nevertheless, we should admit that our model is likely to be bias/specific for male lungs due to the anatomical and physiological differences between males and females. Fourth, our model requires that 11 representative CT slices should be manually selected by an expert. It can be an additional workload for the radiologists, and it has the possibility of incorrect selection of them. The development of a deep learning-based system for detecting anatomic landmarks could be helpful. Fifth, the deep radiomics features were predominantly black box features; therefore, they need to be interpreted by case review and other technical methods. Lastly, the survival prediction performance of the deep radiomics model was not directly compared with that of other various clinical risk-scoring systems such as the Body mass index, airflow Obstruction, Dyspnea, and Exercise (BODE) index, and the incremental value of the deep radiomics model was not fully investigated. In the future, evaluating the relationship between deep features and traditional lung density measurements would be useful and potential added value.

In conclusion, a deep radiomics approach for survival prediction was feasible. The performances of 20 models (top 5 combinations in each size 128, 256, 512, and 1024 of deep features) were compared, and the 
highest C-index of 0.80888008 (95\% CI, 0.7642-0.8373) was obtained by combining 256 features each from a coronal slice and two sagittal slices $(\mathrm{C} 1+\mathrm{S} 1+\mathrm{S} 2)$, as confirmed by concordant results $(\mathrm{C}$-index, $0.7156 ; 95 \%$ CI, 0.7024-0.7288) in an external validation group. The models with 256 deep features performed superior, $\mathrm{C} 1+\mathrm{S} 1+\mathrm{S} 2$ performed best, but there is a risk of false discovery because the differences in the results of different combinations are insignificant.

\section{Data availability}

The datasets generated during and/or analysed during the current study are available from the corresponding author on reasonable request.

Received: 17 September 2020; Accepted: 5 July 2021

Published online: 26 July 2021

\section{References}

1. Halpin, D. M. G. et al. Global initiative for the diagnosis, management, and prevention of chronic obstructive lung disease. Am. J. Respir. Crit. Care Med. 203, 24-36 (2021).

2. Schroeder, J. D. et al. Relationships between airflow obstruction and quantitative CT measurements of emphysema, air trapping, and airways in subjects with and without chronic obstructive pulmonary disease. Am. J. Roentgenol. 201, W460-W470 (2013).

3. Cho, Y. H. et al. Quantitative CT imaging in chronic obstructive pulmonary disease: Review of current status and future challenges. J. Korean Soc. Radiol. 78, 1-12 (2018).

4. Lee, Y. K. et al. Quantitative assessment of emphysema, air trapping, and airway thickening on computed tomography. Lung 186, 157-165 (2008).

5. Diaz, A. A. et al. Relationship of emphysema and airway disease assessed by CT to exercise capacity in COPD. Respir. Med. 104, 1145-1151 (2010).

6. Haruna, A. et al. CT scan findings of emphysema predict mortality in COPD. Chest 138, 635-640 (2010).

7. Cavigli, E. et al. Whole-lung densitometry versus visual assessment of emphysema. Eur. Radiol. 19, 1686-1692 (2009).

8. Lambin, P. et al. Radiomics: Extracting more information from medical images using advanced feature analysis. Eur. J. Cancer 48, 441-446 (2012).

9. Van Griethuysen, J. J. et al. Computational radiomics system to decode the radiographic phenotype. Cancer Res. 77, e104-e107 (2017).

10. Bickelhaupt, S. et al. Prediction of malignancy by a radiomic signature from contrast agent-free diffusion MRI in suspicious breast lesions found on screening mammography. J. Magn. Reson. Imaging 46, 604-616 (2017).

11. Yu, H. et al. Texture analysis as a radiomic marker for differentiating renal tumors. Abdom. Radiol. 42, 2470-2478 (2017).

12. Aerts, H. J. et al. Decoding tumour phenotype by noninvasive imaging using a quantitative radiomics approach. Nat. Commun. 5, 4006 (2014).

13. Oikonomou, A. et al. Radiomics analysis at PET/CT contributes to prognosis of recurrence and survival in lung cancer treated with stereotactic body radiotherapy. Sci. Rep. 8, 4003 (2018).

14. Emaminejad, N. et al. Fusion of quantitative image and genomic biomarkers to improve prognosis assessment of early stage lung cancer patients. IEEE Trans. Biomed. Eng. 63, 1034-1043 (2015).

15. Zhou, Y. et al. CT-based radiomics signature: A potential biomarker for preoperative prediction of early recurrence in hepatocellular carcinoma. Abdom. Radiol. 42, 1695-1704 (2017).

16. Galbán, C. J. et al. Computed tomography-based biomarker provides unique signature for diagnosis of COPD phenotypes and disease progression. Nat. Med. 18, 1711 (2012).

17. Koo, H. J. et al. Prediction of pulmonary function in patients with chronic obstructive pulmonary disease: Correlation with quantitative CT parameters. Korean J. Radiol. 20, 683-692 (2019).

18. Cho, Y. H. et al. Quantitative assessment of pulmonary vascular alterations in chronic obstructive lung disease: Associations with pulmonary function test and survival in the KOLD cohort. Eur. J. Radiol. 108, 276-282 (2018).

19. Krizhevsky, A., Sutskever, I. \& Hinton, G. E. Advances in Neural Information Processing Systems, 1097-1105.

20. Sermanet, P. et al. Overfeat: Integrated recognition, localization and detection using convolutional networks. arXiv preprint arXiv: 1312.6229 (2013).

21. Simonyan, K. \& Zisserman, A. Very deep convolutional networks for large-scale image recognition. arXiv preprint arXiv:1409. $1556(2014)$.

22. Szegedy, C. et al. Going deeper with convolutions. IEEE Conf. Comput. Vis. Pattern Recognit.1-9, 2015.

23. González, G. et al. Disease staging and prognosis in smokers using deep learning in chest computed tomography. Am. J. Respir. Crit. Care Med. 197, 193-203 (2018).

24. Lao, J. et al. A deep learning-based radiomics model for prediction of survival in glioblastoma multiforme. Sci. Rep. 7, 10353 (2017).

25. Li, Z., Wang, Y., Yu, J., Guo, Y. \& Cao, W. Deep learning based radiomics (DLR) and its usage in noninvasive IDH1 prediction for low grade glioma. Sci. Rep. 7, 5467 (2017).

26. Park, T. S. et al. Study design and outcomes of Korean Obstructive Lung Disease (KOLD) cohort study. Tuberc. Respir. Dis. 76, 169-174 (2014).

27. Loh, L.-C., Oh, Y.-M. \& Lee, S.-D. The Asian network for obstructive lung disease (ANOLD)-COPD from an Asian perspective. QJM 108, 921-922 (2015).

28. Loh, L.-C. et al. A novel CT-emphysema index/FEV1 approach of phenotyping COPD to predict mortality. Int. J. Chron. Obstruct. Pulmon. Dis. 13, 2543 (2018).

29. Yoo, J.-W. et al. Comparison of clinico-physiologic and CT imaging risk factors for COPD exacerbation. J. Korean Med. Sci. 26, 1606-1612 (2011).

30. Galiè, N. et al. 2015 ESC/ERS guidelines for the diagnosis and treatment of pulmonary hypertension: The joint task force for the diagnosis and treatment of pulmonary hypertension of the European Society of Cardiology (ESC) and the European Respiratory Society (ERS): Endorsed by: Association for European Paediatric and Congenital Cardiology (AEPC), International Society for Heart and Lung Transplantation (ISHLT). Eur. Heart J. 37, 67-119 (2015).

31. Ishwaran, H., Kogalur, U. B., Blackstone, E. H. \& Lauer, M. S. Random survival forests. Ann. Appl. Stat. 2, 841-860 (2008).

32. Heagerty, P. J., Lumley, T. \& Pepe, M. S. Time-dependent ROC curves for censored survival data and a diagnostic marker. Biometrics 56, 337-344 (2000).

33. Kamarudin, A. N., Cox, T. \& Kolamunnage-Dona, R. Time-dependent ROC curve analysis in medical research: Current methods and applications. BMC Med. Res. Methodol. 17, 1-19 (2017).

34. Kirby, M. et al. Management of COPD: Is there a role for quantitative imaging?. Eur. J. Radiol. 86, 335-342 (2017).

35. Labaki, W. W. et al. The role of chest computed tomography in the evaluation and management of the patient with chronic obstructive pulmonary disease. Am. J. Respir. Crit. Care Med. 196, 1372-1379 (2017). 
36. Johannessen, A. et al. Mortality by level of emphysema and airway wall thickness. Am. J. Respir. Crit. Care Med. 187, 602-608 (2013).

37. Moll, M. et al. Machine learning and prediction of all-cause mortality in COPD. Chest 158, 952-964 (2020).

38. Cho, Y. H. et al. Radiomics approach for survival prediction in chronic obstructive pulmonary disease. Eur. Radiol. https://doi. org/10.1007/s00330-021-07747-7 (2021).

39. Korea National Health and Nutrition Examination Surveys. https://kosis.kr/ (2018).

40. Lim, K. H. et al. Prevalence and factors associated with smoking among adults in Malaysia: Findings from the National Health and Morbidity Survey (NHMS) 2015. Tob. Induc. Dis. 16, 01 (2018).

41. De Torres, J. et al. Sex differences in mortality in patients with COPD. Eur. Respir. J. 33, 528-535 (2009).

42. Barnes, P. J. Sex differences in chronic obstructive pulmonary disease mechanisms. Am. J. Respir. Crit. Care Med. 193, 813-824 (2016).

\section{Acknowledgements}

We thank Mr. Hee Jun Park for data preprocessing. This research was supported by a grant of the Korea Health Technology R\&D Project through the Korea Health Industry Development Institute (KHIDI) funded by the Ministry of Health \& Welfare, Republic of Korea (grant number: HI18C2383), and also supported by the Industrial Strategic Technology Development Program (10072064, Development of Novel Artificial Intelligence Technologies to Assist Imaging Diagnosis of Pulmonary, Hepatic, and Cardiac Diseases and Their Integration into Commercial Clinical PACS Platforms) funded by the Ministry of Trade, Industry and Energy (MI, Korea).

\section{Author contributions}

J.Y.: analysis and interpretation of data, drafting of the article. Y.H.C., S.M.L., J.H.: data curation and oversight, conceptual feedback. J.S.L., Y.M.O., S.D.L., L.C.L., C.K.O.: data provision. J.B.S. and N.K.: study conception and design, critical revision of the article for important intellectual content, and final approval of the article.

\section{Competing interests}

The authors declare no competing interests.

\section{Additional information}

Supplementary Information The online version contains supplementary material available at https:/doi.org/ 10.1038/s41598-021-94535-4.

Correspondence and requests for materials should be addressed to J.B.S. or N.K.

Reprints and permissions information is available at www.nature.com/reprints.

Publisher's note Springer Nature remains neutral with regard to jurisdictional claims in published maps and institutional affiliations.

(c) (i) Open Access This article is licensed under a Creative Commons Attribution 4.0 International License, which permits use, sharing, adaptation, distribution and reproduction in any medium or format, as long as you give appropriate credit to the original author(s) and the source, provide a link to the Creative Commons licence, and indicate if changes were made. The images or other third party material in this article are included in the article's Creative Commons licence, unless indicated otherwise in a credit line to the material. If material is not included in the article's Creative Commons licence and your intended use is not permitted by statutory regulation or exceeds the permitted use, you will need to obtain permission directly from the copyright holder. To view a copy of this licence, visit http://creativecommons.org/licenses/by/4.0/.

(C) The Author(s) 2021 